\title{
Diesel Exhaust Particles Impair Therapeutic Effect of Human Wharton's Jelly-Derived Mesenchymal Stem Cells against Experimental Colitis through ROS/ERK/cFos Signaling Pathway
}

\author{
Hyun Sung Park ${ }^{1,2, *}$, Mi-Kyung $\mathrm{Oh}^{3,4, *}$, Joong Won Lee ${ }^{5}$, Dong-Hoon Chae ${ }^{3}$, Hansol Joo ${ }^{1,2}$, \\ Ji Yeon Kang, ${ }^{1,2}$, Hye Bin $\mathrm{An}^{3}$, Aaron $\mathrm{Yu}^{3}$, Jae Han Park ${ }^{3}$, Hee Min Yoo ${ }^{6,7}$, Hyun Jun Jung ${ }^{8}$, \\ Uimook Choi ${ }^{9}$, Ji-Won Jung, In-Sook Kim ${ }^{1,2}$, Il-Hoan Oh ${ }^{1,10}$, Kyung-Rok Yu ${ }^{3}$
}

\begin{abstract}
${ }^{l}$ Department of Biomedicine and Health Sciences, College of Medicine, The Catholic University of Korea, Seoul, Korea, ${ }^{2}$ Department of Medical Life Sciences, College of Medicine, The Catholic University of Korea, Seoul, Korea, ${ }^{3}$ Department of Agricultural Biotechnology and Research Institute of Agriculture and Life Sciences, Seoul National University, Seoul, Korea, ${ }^{4}$ Bio-MAX Institute, Seoul National University, Seoul, Korea, ${ }^{5}$ Division of Allergy and Respiratory Disease Research, Department of Chronic Disease Convergence Research, Korea National Institute of Health, Cheongju, Korea, ${ }^{6}$ Biometrology Group, Korea Research Institute of Standards and Science (KRISS), Daejeon, Korea, ${ }^{7}$ Department of Bio-Analytical Science, University of Science and Technology (UST), Daejeon, Korea, ${ }^{8}$ Division of Nephrology, Department of Medicine, Fohns Hopkins University School of Medicine, Baltimore, MD, USA, ${ }^{9}$ Laboratory of Clinical Immunology and Microbiology, NIAID, NIH, Bethesda, MD, USA, ${ }^{10}$ Catholic High-Performance Cell Therapy Center \& Department of Medical Life Science, College of Medicine, The Catholic University of Korea, Seoul, Korea
\end{abstract}

Background and Objectives: Epidemiological investigations have shown positive correlations between increased diesel exhaust particles (DEP) in ambient air and adverse health outcomes. DEP are the major constituent of particulate atmospheric pollution and have been shown to induce proinflammatory responses both in the lung and systemically. Here, we report the effects of DEP exposure on the properties of human Wharton's jelly-derived mesenchymal stem cells (WJ-MSCs), including stemness, regeneration, and immunomodulation.

Methods and Results: Non-apoptotic concentrations of DEP $(10 \mu \mathrm{g} / \mathrm{ml})$ inhibited the migration and osteogenic differentiation capacity of WJ-MSCs. Gene expression profiling showed that DEP increased intracellular reactive oxygen species (ROS) and expression of pro-inflammatory and metabolic-process-related genes including cFos. Furthermore, WJ-MSCs cultured with DEP showed impaired suppression of T cell proliferation that was reversed by inhibition of ROS or knockdown of cFos. ERK inhibition assay revealed that DEP-induced ROS regulated cFos through activation of ERK but not NF- $\kappa$ B signaling. Overall, low concentrations of DEP $(10 \mu \mathrm{g} / \mathrm{ml})$ significantly suppressed the stemness and immunomodulatory properties of WJ-MSCs through ROS/ERK/cFos signaling pathways. Furthermore, WJ-MSCs cultured with DEP impaired the therapeutic effect of WJ-MSCs in experimental colitis mice, but was partly reversed by inhibition of ROS.

Conclusions: Taken together, these results indicate that exposure to DEP enhances the expression of pro-inflammatory cytokines and immune responses through a mechanism involving the ROS/ERK/cFos pathway in WJ-MSCs, and that DEP-induced ROS damage impairs the therapeutic effect of WJ-MSCs in colitis. Our results suggest that modulation of ROS/ERK/cFos signaling pathways in WJ-MSCs might be a novel therapeutic strategy for DEP-induced diseases.

Keywords: Diesel exhaust particle, Mesenchymal stem cell, Oxidative stress, ERK, cFos, Immunomodulation

Received: October 6, 2021, Revised: November 10, 2021, Accepted: November 10, 2021, Published online: December 31, 2021 Correspondence to Kyung-Rok Yu

Department of Agricultural Biotechnology and Research Institute of Agriculture and Life Sciences, Seoul National University, Seoul 08826, Korea

Tel: +82-2-880-4807, Fax: +82-2-873-2271, E-mail: cellyu@snu.ac.kr

Co-Correspondence to Il-Hoan Oh

Department of Biomedicine and Health Sciences, College of Medicine, The Catholic University of Korea, Seoul 06591, Korea

Tel: +82-2-2258-8265, Fax: +82-2-591-3994, E-mail: iho@catholic.ac.kr

*These authors contributed equally to this work.

(c) This is an open-access article distributed under the terms of the Creative Commons Attribution Non-Commercial License (http://creativecommons.org/ licenses/by-nc/4.0/), which permits unrestricted non-commercial use, distribution, and reproduction in any medium, provided the original work is properly cited. Copyright (c) 2022 by the Korean Society for Stem Cell Research 


\section{Introduction}

Air pollution is one of the most concerning environmental risks to human society. The primary source of air pollutants is particulate matter $(\mathrm{PM})$, which is categorized into three types according to particle size: coarse $(<10 \mu \mathrm{m})$, fine $(<2.5 \mu \mathrm{m})$, and ultrafine $(<0.2 \mu \mathrm{m})$. The majority of particles contributing to airborne PM is ultrafine diesel exhaust particles (DEP) that are emitted from diesel engines (1).

DEP is a complex mixture of organic and inorganic material including polycyclic aromatic hydrocarbons (PAHs). There is increasing evidence to suggest that oxidative stress caused by PAH-induced reactive oxygen species (ROS) affects macrophages and epithelial cells and is the main driver of inflammation and damage in the lungs (2).

When alveolar macrophages and bronchial epithelial cells first encounter inhaled DEP, DEP trigger activation of macrophages to the $\mathrm{Ml}$ phenotype and release of pro-inflammatory cytokines (3). Furthermore, DEP activate mitogen-activated protein kinases (MAPKs) and nuclear factor- $\kappa \mathrm{B}(\mathrm{NF}-\kappa \mathrm{B})$ to induce IL-6, IL-8, and GM-CSF, which are markers for pro-inflammation (4). Recent studies suggest that DEP also affects $T$ cell differentiation. In a mouse model of multiple sclerosis, the aryl hydrocarbon receptor (AHR) regulates development of regulatory $\mathrm{T}$ cells (Treg) and T helper 17 (Th17) cells (5). Taken together, these findings indicate that DEP-induced PAHs enhance oxidative stress and activate pro-inflammatory responses in macrophages, bronchial epithelial cells, and naïve $\mathrm{T}$ cells.

Mesenchymal stem cells (MSCs) have been reported to modulate immune responses to tissue damage and inflammation by secreting numerous bioactive molecules, cytokines, growth factors, and chemokines and by modulating cell-to-cell contact (6). As a result of their hypoimmunogenic and immunosuppressive properties, MSCs show potential to modulate severe inflammatory responses (7). MSCs suppress proliferation of $\mathrm{CD} 4+, \mathrm{CD} 8+$, and proinflammatory type 1 helper $\mathrm{T}$ cells (Th1) to inhibit hyper-adaptive immune reactions (8). MSCs also inhibit proinflammatory M1 macrophages and activate anti-inflammatory M2 macrophages (9). Previously, we have shown that the human MSC secretome, including transforming growth factor-beta (TGF- $\beta$ ), prostaglandin E2 (PGE2), indoleamine 2,3-dioxygenase (IDO), and extracellular vesicles (EVs), is responsible for the anti-inflammatory properties $(10,11)$. The mechanisms and therapeutic effects of MSCs in autoimmune and DEP-induced diseases have been studied; however, neither the nature of direct interactions between DEP and MSCs nor the precise mechanism of DEP effects on MSCs have been elucidated (12-14).

In this study, we investigated the detailed mechanisms of DEP effects on MSC stemness, immunomodulatory functions, and pro-inflammatory activation signal pathways.

\section{Materials and Methods}

\section{Diesel exhaust particle preparation}

The Standard Reference Material (SRM) 1650b diesel particulate matter was obtained from the National Institute of Standards and Technology (NIST, Gaithersburg, MD, USA). The DEP were suspended in dimethyl sulfoxide as a $10 \mathrm{mg} / \mathrm{ml}$ stock solution.

\section{Human Wharton's jelly-derived mesenchymal stem cells preparation and culture}

Wharton's jelly-derived mesenchymal stem cells (WJMSCs) were isolated from umbilical cords of healthy fullterm babies as previously described (10). The procedures for tissue harvesting and obtaining informed consent were approved by the Asan Medical Center Institutional Review Board (Protocol no. 2015-3030). WJ-MSCs were cultured in Dulbecco's Modified Eagle Medium (DMEM; Gibco, Grand Island, NY, USA) supplemented with $10 \%$ fetal bovine serum (FBS; Tissue Culture Biologicals, Tulare, CA, USA), 1\% Glutamax (Gibco), 1\% antibiotic/antimycotic solution (Gibco), $25 \mathrm{ng} / \mathrm{ml}$ epidermal growth factor (EGF; Peprotech, Rocky Hill, NJ, USA), and $50 \mathrm{ng} / \mathrm{ml}$ basic fibroblast growth factor (bFGF; Peprotech) in a humidified atmosphere containing $5 \% \mathrm{CO}_{2}$ at $37^{\circ} \mathrm{C}$. Cells were passaged every $3 \sim 4$ days using $0.05 \%$ trypsin/EDTA. The cells were pretreated with N-acetyl-1-cysteine (NAC; 5 mM; Sigma-Aldrich, St. Louis, MO, USA), U0126 (10 $\mu \mathrm{M}$; Cell Signaling Technology, Danvers, MA, USA), or parthenolide (PTL; $5 \mu \mathrm{M}$; Sigma-Aldrich) before stimulation with DEP $(10 \mu \mathrm{g} / \mathrm{ml})$ as indicated.

\section{MTT assay}

The 3-(4,5-dimethylthiazol-2-yl)-2,5-diphenyltetrazolium bromide (MTT; Sigma-Aldrich) assay was performed to assess the effect of DEP on the viability of WJ-MSCs. WJ-MSCs were seeded into a 24-well plate at $5 \times 10^{4}$ cells/well with and without various concentrations of DEP for $48 \mathrm{hr}$. MTT reagent $(0.5 \mathrm{mg} / \mathrm{ml}$; Sigma-Aldrich) was added, and the cells were cultured for $3 \mathrm{hr}$ at $37^{\circ} \mathrm{C}$. The resulting formazan precipitate was solubilized in $0.04 \mathrm{M}$ $\mathrm{HCl}$, and the absorbance was measured at $570 \mathrm{~nm}$ using a Synergy ${ }^{\mathrm{TM}} \mathrm{H}$ l microplate reader (Biotek, Winooski, WT, USA). 


\section{Apoptosis assay}

For apoptosis assessment, WJ-MSCs were seeded into a 96-well plate at $1 \times 10^{4}$ cells/well with $\operatorname{DEP}(0 \sim 40 \mu \mathrm{g} / \mathrm{ml})$ for $48 \mathrm{hr}$. The cells were stained with Annexin-V-FITC (BD Biosciences, Franklin Lakes, NJ, USA) and 7-AAD (BD Biosciences) following the manufacturer's instructions and analyzed by flow cytometry.

\section{Scratch wound healing assay}

WJ-MSCs were seeded into a 24 -well plate at $1.5 \times 10^{5}$ cells/well and cultured overnight to $100 \%$ confluency. The cells were incubated with $10 \mu \mathrm{g} / \mathrm{ml}$ mitomycin C (MMC; Sigma-Aldrich) for $1 \mathrm{hr}$ to render them mitotically inactive. The cells were scratched with a 200 ul pipette tip and treated with $0 \sim 10 \mu \mathrm{g} / \mathrm{ml}$ DEP. The cells migrating into the scratched area were photographed at 0,18 , and $36 \mathrm{hr}$, and migration distances were calculated using Image J software (National Institutes of Health, Bethesda, MD, USA).

\section{In vitro differentiation assay}

WJ-MSCs were seeded into a 12 -well plate at $5 \times 10^{4}$ cells/well and incubated with various concentrations of DEP for $48 \mathrm{hr}$. When the cells reached $70 \sim 80 \%$ confluence, StemMACS ${ }^{\mathrm{TM}}$ Adipodiff Media and OsteoDiff Media (Miltenyi Biotec, Bergisch Gladbach, Germany) were added to assess adipogenic and osteogenic differentiation, respectively. The induction media were changed every three days. After three weeks, calcium deposits and lipid droplets were stained with Alizarin Red S (Sigma-Aldrich) and Oil Red O (Abcam, Cambridge, UK), respectively. For quantification, Alizarin-Red-S-stained cells were dissolved in $100 \mathrm{mM}$ cetylpyridinium chloride (Sigma-Aldrich), and the absorbance was measured at 590 $\mathrm{nm}$. Oil-Red-O-stained cells were dissolved in 100\% isopropanol, and the absorbance was measured at $490 \mathrm{~nm}$ using a spectrophotometer.

\section{RNA isolation and sequencing (RNA-seq)}

RNA sequencing (RNA-seq) was performed by Bioneer Co. (Daejeon, Korea) using Illumina technology. Total RNA was extracted from the WJ-MSCs incubated with or without $5 \mu \mathrm{g} / \mathrm{ml}$ of DEP using TRIzol reagent (Invitrogen, Carlsbad, CA, USA). The mRNA sequencing library was generated using the Illumina TruSeq strand mRNA sample preparation kit (Illumina, San Diego, CA, USA) and sequenced using NovaSeq $6000(2 \times 150$ paired end sequencing, Illumina) according to the manufacturer's protocol. After removing the adapter sequence and filtering the low-quality readings using an in-house script, the filtered readings were mapped to the reference human genome using TopHat (15). For differential expression analysis, the gene expression of each group was quantified using cufflink v2.1.1 (16) by mapping the readings to the human gene annotation database (hg19). Next, the differentially expressed genes (DEGs) in the control and DEP-treated WJ-MSCs were identified based on absolute $\log 2$-fold change $\geq 1$ and $\mathrm{p}<0.05$ using Cuffdiff $(17,18)$. Then, heatmaps were generated using an in-house script, and clustering analysis was performed using a hierarchical clustering method. Volcano plots of DEGs were prepared using GraphPad Prism software (version 8.0.1; GraphPad Software, San Diego, CA, USA).

\section{Gene ontology (G0) analysis and gene set enrichment analysis (GSEA)}

We identified gene sets based on gene ontology (GO) into three categories: biological processes (BP), cellular components (CC), and molecular function (MF). The significance of the gene sets was calculated using gene set enrichment analysis (GSEA v3.0, https:/www.gsea-msigdb. org/gsea/index.jsp). GO-based trend testing was performed using Fisher's exact test.

\section{Quantitative real-time PCR}

Total RNA was extracted using TRIzol reagent (Invitrogen), and $2 \mu \mathrm{g}$ of total RNA was converted to cDNA using Superscript ${ }^{\mathrm{TM}}$ III reverse transcriptase (Invitrogen). Realtime PCR was performed using the SYBR Green Master Mix (Applied Biosystems, Foster City, CA, USA) and measured using a MX3000P thermal cycler (Agilent Technologies, Santa Clara, CA, USA). $\beta$-actin was used as the reference gene for normalization. The primer sequences for qRT-PCR are listed in Supplementary Table S1.

\section{Detection of intracellular reactive oxygen species (ROS)}

Intracellular reactive oxygen species (ROS) were detected using the peroxide-sensitive fluorophore 2',7'-dichlorofluorescin diacetate (DCFDA, Sigma-Aldrich). WJ-MSCs were incubated with various concentrations of DEP for 48 hr. After incubation, cells were washed with PBS and incubated with $10 \mu \mathrm{M}$ DCFDA in serum-free culture medium for $30 \mathrm{~min}$ at $37^{\circ} \mathrm{C}$. The mean DCFDA fluorescence was analyzed using an Attune NxT flow cytometer (Thermo Fisher Scientific, Waltham, MA, USA). 


\section{Isolation of umbilical cord blood-derived mononuclear cells}

Umbilical cord blood (UCB) samples were obtained from the Catholic Hematopoietic Stem Cell Bank (CHSCB) under approval of Institutional Review Board (IRB) of the Seoul National University (IRB No. E2011/001-010). Human mononuclear cells (MNCs) were isolated by Ficoll gradient centrifugation as previously described (10), and were cultured in RPMI 1640 medium (Gibco) supplemented with $10 \%$ FBS.

\section{T cell proliferation assay}

WJ-MSCs were pre-cultured with or without NAC for $1 \mathrm{hr}$, and then various concentrations of DEP were added for $48 \mathrm{hr}$. Subsequently, the WJ-MSCs were treated with $10 \mu \mathrm{g} / \mathrm{ml} \mathrm{MMC} \mathrm{for} 1 \mathrm{hr}$ to arrest cell proliferation, and the cells were seeded at $1 \times 10^{4}$ cells per well in a 96-well plate. After $24 \mathrm{hr}, \mathrm{MNCs}$ were labeled with $2 \mu \mathrm{M}$ 5,6-carboxyfluorescein succinimidyl ester (CFSE; Thermo Fisher Scientific), and $1 \times 10^{5} \mathrm{MNCs}$ were added to each well containing WJ-MSCs, in the presence of anti-CD3/CD28 microbeads (Gibco) and recombinant human IL-2 $(30 \mathrm{U} / \mathrm{ml}$; PeproTech). After six days of incubation, the cells were stained with fluorescence-labeled human monoclonal antibodies against CD45-APC-H7, CD3-BV510, CD4-APC, and CD8-BV421 (BD Biosciences). Proliferation of total T cells and $\mathrm{T}$ cell subpopulations was measured by dilution of CFSE using a FACScanto flow cytometer (BD Biosciences). Viable lymphocytes were gated on 7AADnegative cells.

\section{WJ-MSCs small interfering RNAs (siRNA) transfection}

To silence the expression of $c F o s$, WJ-MSCs were transiently transfected with specific siRNAs (Bioneer) using the Lipofectamine ${ }^{\mathrm{TM}}$ RNAiMAX reagent (Invitrogen) according to the manufacturer's protocol. After $4 \mathrm{hr}$ of transfection, $10 \mu \mathrm{g} / \mathrm{ml}$ of DEP was added, and the cells were incubated at $37^{\circ} \mathrm{C}$ for $24 \mathrm{hr}$.

\section{Western blot analysis}

The cells were lysed using RIPA buffer (Thermo Fisher Scientific), and the protein concentrations were determined using a BCA assay kit (Thermo Fisher Scientific). Equal amounts of proteins were obtained after SDS-PAGE separation and analyzed using primary antibodies against $\mathrm{cFos}$, ERK, p-ERK, and $\mathrm{p}-\mathrm{I} \kappa \mathrm{B} \alpha$ (Cell Signaling Technology), and GAPDH (Santa Cruz Biotechnology, Dallas, TX, USA). The bands were visualized using an enhanced chemiluminescence assay kit (Thermo Fisher Scientific) and luminescent image analyzer (LAS-3000 system;
Fujifilm, Tokyo, Japan). For quantification, ImageJ software (National Institutes of Health) were used to analyzed the bands intensity.

\section{Dextran sulfate sodium (DSS)-induced colitis mice}

All animal experiments were carried out in accordance with the approved by the Institutional Animal Care and Use Committee (IACUC) of Seoul National University (protocol no. SNU-201021-2-1). Colitis was induced by administration of $3 \%$ dextran sulfate sodium (DSS; MP Biomedicals, Santa Ana, CA, USA) in drinking water for 7 days. 8-week-old C57BL/6 mice were randomly assigned to 5 groups ( $\mathrm{n}=10$ per group: (1) Negative control, (2) DSS only, (3) DSS with WJ-MSC, (4) DSS with DEP-treated WJ-MSC (DEP group), (5) DSS with NAC pretreated DEP-treated WJ-MSC (NAC group). Mice were injected intraperitoneally with PBS or cells $\left(2 \times 10^{6}\right.$ cells in $200 \mu$ l) on day 1 after DSS drinking. The severity of colitis was assessed daily using the disease activity index (DAI). All the mice were sacrificed on day 12 and their colon lengths and weights were measured. Myeloperoxidase (MPO) activity assay and histopathological evaluation were performed as previously described (11).

\section{Statistical analysis}

For statistical analysis, all experiments were performed at least in triplicate. Where data were normally distributed, the significance was determined using Student's t-test or a one-way analysis of variance (ANOVA) followed by Tukey's multiple comparisons test. The data are presented as the mean \pm standard deviation. All statistical analyses were performed using GraphPad Prism software (version 8.0.1).

\section{Results}

\section{Diesel exhaust particles (DEP) impair WJ-MSC functions}

Many studies have shown that DEP activate pro-inflammatory responses and affect various cellular functions in diverse cell types (19-22). We therefore investigated the effects of DEP on stemness and immunomodulatory functions of WJ-MSCs. First, we assessed DEP-induced toxicity using the MTT assay; incubation with $10 \mu \mathrm{g} / \mathrm{ml}$ or lower concentrations of DEP did not affect the cell viability, but the viability was significantly reduced at $20 \mu \mathrm{g} / \mathrm{ml}$ and $40 \mu \mathrm{g} / \mathrm{ml}$ DEP (Fig. 1a). Moreover, higher concentrations of DEP significantly induced early and late apoptosis (Fig. 1b). Based on these results, non-apoptotic concentrations of DEP $(0 \sim 10 \mu \mathrm{g} / \mathrm{ml})$ were used in subsequent experiments. 


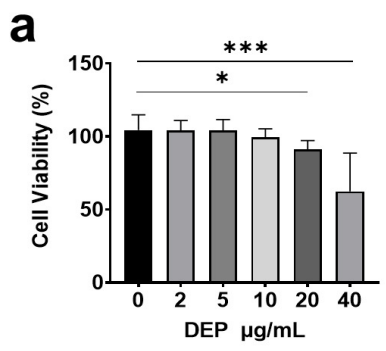

b
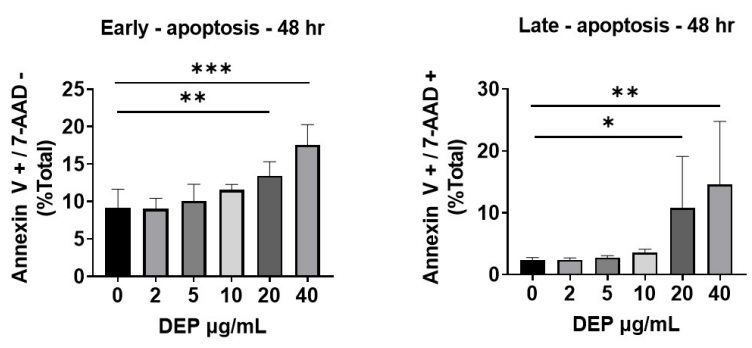

C DEP
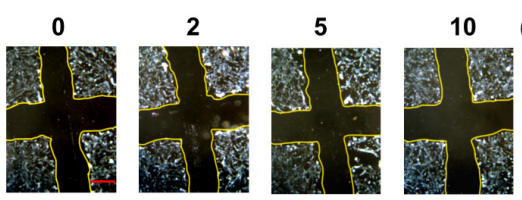

$(\mu \mathrm{g} / \mathrm{ml})$

$18 \mathrm{hr}$
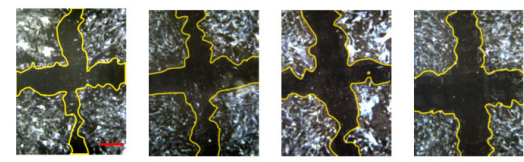

$36 \mathrm{hr}$
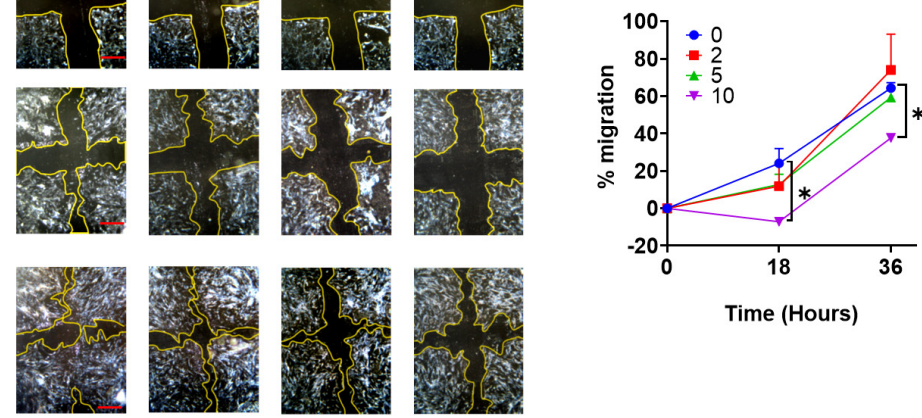

d
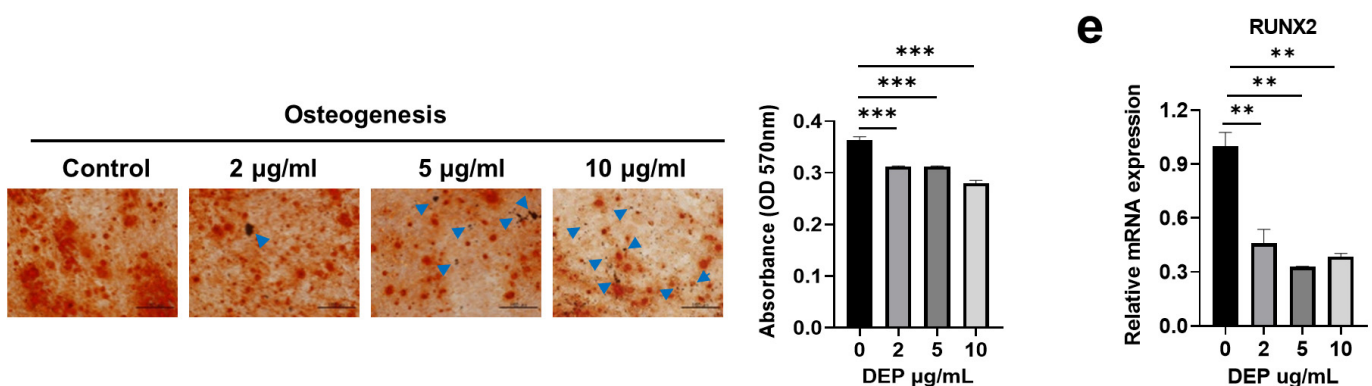

Fig. 1. Diesel exhaust particles (DEP) affect WJ-MSC function. (a, b) The WJ-MSCs were treated with DEP $(0 \sim 40 \mu \mathrm{g} / \mathrm{ml})$ for $48 \mathrm{hr}$. (a) Cell viability was assessed using an MTT assay. (b) Early and late apoptosis were measured by labeling with Annexin-V-FITC and 7-AAD for flow cytometry analysis. (c) A scratched wound healing assay of DEP-treated WJ-MSCs $(0 \sim 10 \mu \mathrm{g} / \mathrm{ml})$ was performed to analyze cell migration into the scratched area. After 18 and 36 hr DEP treatment, migration into the scratched area was measured. (Scale bar: 200 $\mu \mathrm{m})$. (d, e) DEP-treated WJ-MSCs were cultured with osteogenic differentiation medium for 21 days. (d) Representative light microscopic images of osteocytes. Calcium deposits in cells cultured under osteogenesis were stained with Alizarin Red S (scale bar: $100 \mu \mathrm{m}$ ) and quantified by measuring absorbance using a spectrophotometer. Blue arrows indicate DEP. (e) Expression of an osteogenic gene (RUNX2) was quantified by qRT-PCR with $\beta$-actin as the reference gene. Data are presented as the mean \pm S.D. of triplicate experiments $\left({ }^{*} p<0.05\right.$; $\left.{ }^{* *} \mathrm{p}<0.01 ; * * * \mathrm{p}<0.001\right)$.

To test the migration and differentiation potential of DEP-treated WJ-MSCs, a scratch wound assay was performed, and in vitro differentiation (adipogenesis and osteogenesis) was assessed. The cell-based wound healing assay showed that migration of WJ-MSCs incubated with 10 $\mu \mathrm{g} / \mathrm{ml}$ DEP was significantly reduced compared with that of control WJ-MSCs (Fig. 1c). When cultured under osteogenesis differentiation conditions, calcium deposition was reduced by incubation with DEP in a concentration-dependent manner (Fig. 1d), as was the expression of the osteogenic differentiation marker RUNX2 (Fig. 1e). However, there were no significant effects on adipogenic differentiation at any DEP concentration tested (Supplementary Fig. 1a and 1b). These results indicate that DEP inhibits migration and osteogenic differentiation of WJ-MSCs.

\section{DEP increases ROS production and pro-inflammatory cytokine expression in WJ-MSCs}

RNA sequencing (RNA-seq) was performed to examine changes in the gene expression profile of DEP-treated 
WJ-MSCs. Global differences in gene expression profiles were visualized by the hierarchical clustering heat map and the volcano plot. Clustering analyses showed 504 differentially expressed genes (DEG) in control MSCs and DEP-treated WJ-MSCs (Fig. 2a). These genes were used in gene ontology (GO)-term analyses to identify overrepresented biological functions. The enriched GO-catego- ries identified in DEP-treated WJ-MSCs included cellular processes, metabolic processes, and biological regulation (Supplementary Fig. 2a and 2b). Among the most upregulated genes related to metabolic processes in DEP-treated WJ-MSCs, we focused on $c F o s$, as it provides a possible link to DEP-induced pathology (Fig. 2b). We confirmed that $c F o s$ mRNA expression was significantly increased af- a

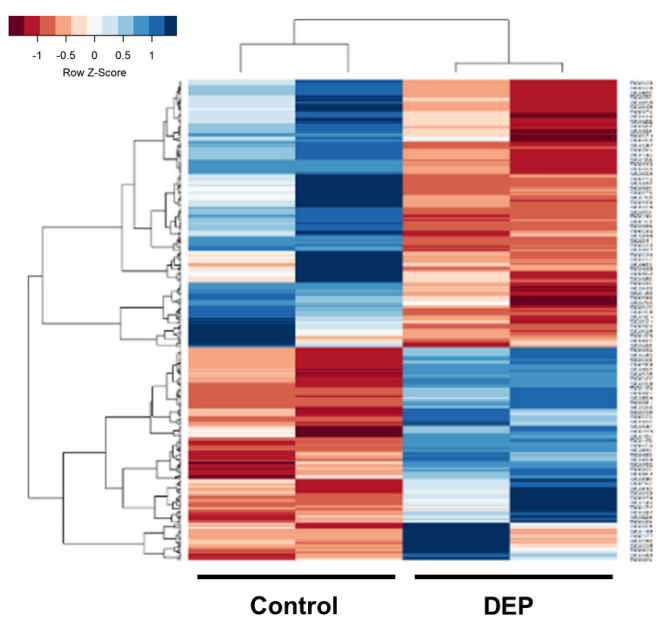

b

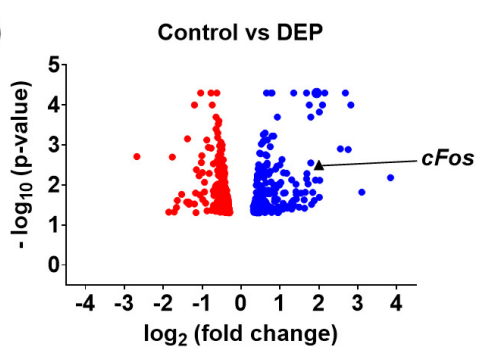

C

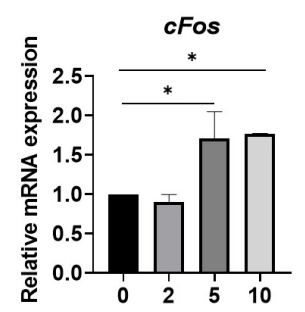

d

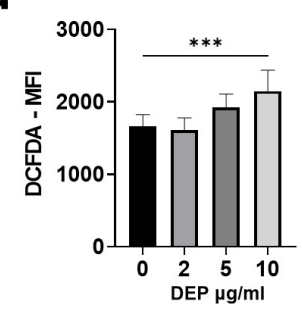

e

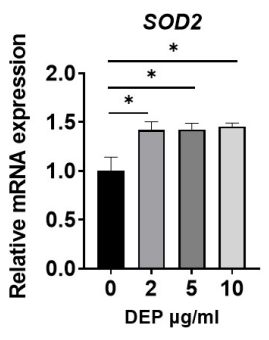

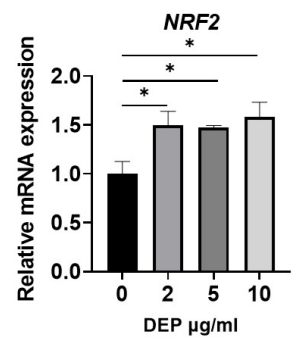

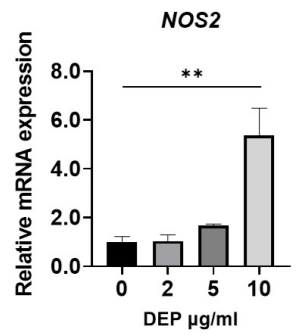

f

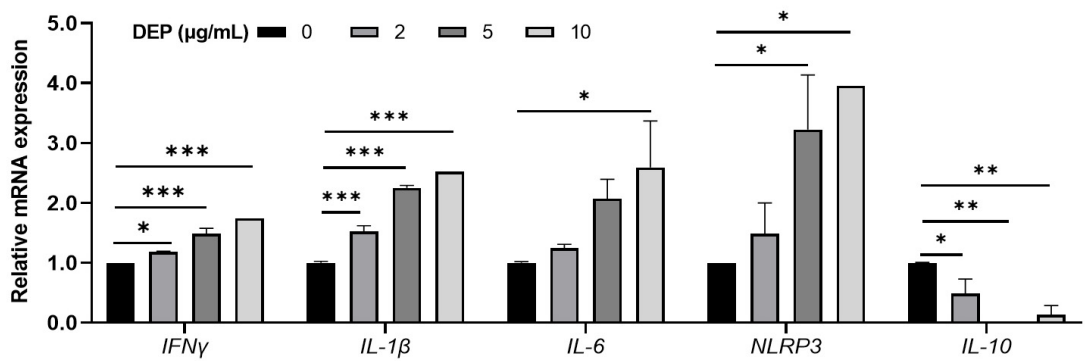

Fig. 2. DEP-treated WJ-MSCs express ROS and pro-inflammatory genes. (a) Heatmap of hierarchical clustering. Differentially expressed gene (DEG) profile for the control and DEP-treated WJ-MSCs were analyzed (p-value $<0.05$ ). (b) Volcano plot highlighting DEGs for the control and DEP-treated WJ-MSCs and notable genes of interest. The blue dots represent significantly upregulated genes, and the red dots represent significantly downregulated genes ( $|\log 2 \mathrm{FC}| \geq 1$ and FDR $<0.01$ ). (c) Transcription of $c F$ os was quantified by qRT-PCR. (d) The WJ-MSCs were incubated with DEP at the indicated concentrations for $6 \mathrm{hr}$. Intracellular ROS levels were measured using the ROS-sensitive fluorophore $2^{\prime}, 7^{\prime}$-dichlorofluorescin diacetate (DCFDA) and analyzed by flow cytometry. (e) Transcription of ROS-related genes was determined by qRT-PCR and normalized to $\beta$-actin expression. ( $\mathrm{f}$ ) Transcription of inflammation-related genes was determined by qRT-PCR and normalized to $\beta$-actin expression. The data are presented as the mean \pm S.D. of three independent experiments $\left({ }^{*} \mathrm{p}<0.05 ;{ }^{* *} \mathrm{p}<0.01 ;{ }^{* * *} \mathrm{p}\right.$ $<0.001$ ). 
ter incubation of WJ-MSCs with DEP (Fig. 2c).

As DEP-induced pathology is largely due to ROS-mediated cellular toxicity, we assessed induction of ROS in DEP-treated WJ-MSCs using DCFDA staining. At $10 \mu$ $\mathrm{g} / \mathrm{ml}$ DEP, intracellular ROS increased significantly (Fig. 2d), and expression of ROS-related genes SOD2, Nrf2, and NOS2 was increased (Fig. 2e). We next investigated the regulation of inflammatory genes by DEP in WJ-MSCs. Expression of pro-inflammatory genes IFN $\gamma$, IL-1 $\beta$, $I L-6$, and NLRP3 was significantly upregulated by DEP treatment, and the anti-inflammatory cytokine $I L-10$ was downregulated (Fig. 2f). These results indicate that DEP upregulates ROS-related genes and pro-inflammatory genes in WJ-MSCs.

\section{DEP inhibits immunomodulatory properties of WJ-MSCs via ROS}

To determine whether DEP-induced ROS are implicated in the immunosuppressive properties of WJ-MSCs, we performed a $\mathrm{T}$ cell proliferation assay in the presence of DEP and an ROS inhibitor (N-acetyl-l-cysteine, NAC). DEPtreated WJ-MSCs with or without added NAC were co-cultured with CFSE-labeled human umbilical cord blood-derived mononuclear cells that were activated with CD3/ CD28 Dynabeads and IL-2. DEP inhibited WJ-MSCmediated reduction of the proliferation of total lymphocytes and of CD4+ and CD8 + T lymphocytes in a concentration-dependent manner. This inhibitory effect was significantly attenuated by the ROS inhibitor (Fig. 3a and 3b, Supplementary Fig. 3a). The percentage of MNCs per cell division shifted to later cycles (cycle 4, 5) depending on the concentration of DEP and returned to the previous cycle (cycle 2, 3) when the WJ-MSCs were incubated with NAC (Fig. 3c, Supplementary Fig. 3b). Taken together, these data indicate that DEP-induced ROS are a major inhibitor of the immunosuppressive effects of WJ-MSCs.

\section{cFos is involved in inhibition of immunomodulatory function of DEP-treated WJ-MSCs}

A previous study showed that $\mathrm{cFos}$ is a direct downstream target of ROS and is considered an ROS-induced pro-inflammatory gene (23). Therefore, we investigated whether ROS are implicated in DEP-induced $c F o s$ in WJ-MSCs. ROS inhibitor treatment significantly inhibited DEP-induced $c F o s$ mRNA and protein expression in WJ-MSCs (Fig. 3d and 3e). To investigate involvement of cFos in the immunomodulatory effects of WJ-MSCs, WJ-MSCs were transfected with specific siRNAs against $c F o s$ and cultured in the presence of $10 \mu \mathrm{g} / \mathrm{ml}$ of DEP. We confirmed siRNA-mediated knockdown of cFos at both the mRNA and protein levels in DEP-treated WJ-MSCs (Fig. 4a and 4b). Knockdown of cFos reversed the increase in $\mathrm{T}$ cell proliferation in DEP-treated WJ-MSCs (Fig. 4c and 4d, Supplementary Fig. 4a). As indicated in Fig. 4e, the percentages of MNCs per cell division in cFos-knockdown WJ-MSCs increased in the 2nd and 3rd division cycles compared with DEP-treated WJ-MSCs (Fig. 4e, Supplementary Fig. 4b). In DEP-treated cFos-depleted WJ-MSCs, the osteogenic potential was markedly greater than in DEP-treated control WJ-MSCs (Fig. 4f). In addition, up-regulated pro-inflammatory cytokines, such as $I L-1 \beta, I L-6$, and NLRP3, in DEP-treated WJ-MSCs were decreased after $c F o s$ depletion whereas the anti-inflammatory cytokine IL-10 was increased (Fig. 4g). These results indicate that DEP inhibits the immunomodulatory function of WJ-MSCs through cFos signaling.

\section{DEP activates cFos through ERK signaling}

Increased concentrations of proinflammatory cytokines in human airway epithelial cells after DEP exposure have been reported to be associated with activation of NF- $\kappa \mathrm{B}$, cFos, and MAPK (24). Similarly, DEP increased phosphorylation of ERK and $\mathrm{I} \kappa \mathrm{B} \alpha$ in a concentration-dependent manner in WJ-MSCs (Fig. 5a). To examine whether ERK and NF- $\kappa \mathrm{B}$ activation is involved in DEP-induced cFos signaling, WJ-MSCs were pre-treated with the ERK kinase inhibitor U0126 and the NF- $\kappa$ B inhibitor parthenolide (PTL). The U0126 and PTL almost completely blocked the activation of ERK and $\mathrm{NF}-\kappa \mathrm{B}$, respectively (Fig. 5b), and U0126 but not PTL significantly inhibited DEP-induced cFos expression (Fig. 5c). Taken together, these results indicate that inhibition of the immunomodulatory function of WJ-MSCs by DEP is mediated through $\mathrm{ROS} / \mathrm{ERK} / \mathrm{cFos}$ signaling.

\section{DEP-induced ROS impairs the protective effect of WJ-MSCs against DSS-induced colitis in mice}

To evaluate whether DEP-induced ROS affected the therapeutic effect of WJ-MSCs in DSS-induced colitis, WJ-MSCs cultured with DEP and NAC were administered to mice 1 day after colitis induction (Fig 6a). As shown in Fig. 6a and 6b, mice in the DEP group displayed continuous body weight loss and remarkably elevated disease activity index (DAI). However, the NAC group was significantly ameliorated body weight loss and DAI as well as increased the survival rate (Fig. $6 \mathrm{a} \sim \mathrm{c})$. Simultaneously, the colons of the DEP group were obviously shortened when compared to the WJ-MSC group whereas NAC group significantly increased the colon length and de- 
a

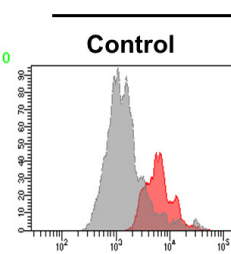

DEP

b
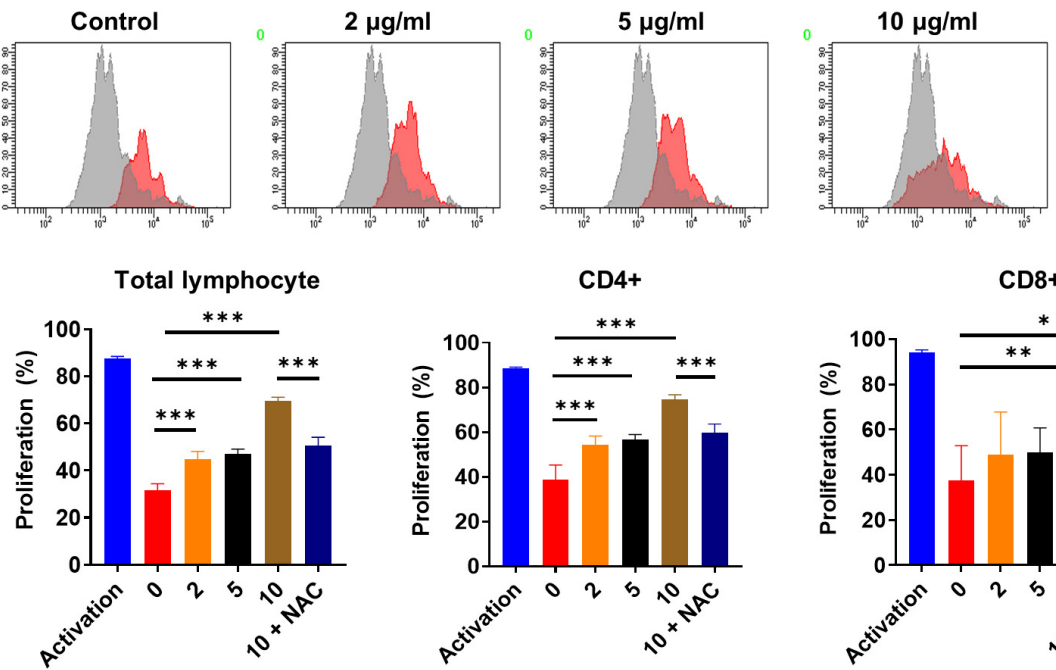

$10 \mu \mathrm{g} / \mathrm{ml}+\mathrm{NAC}$

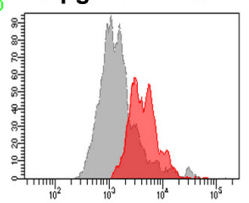

b
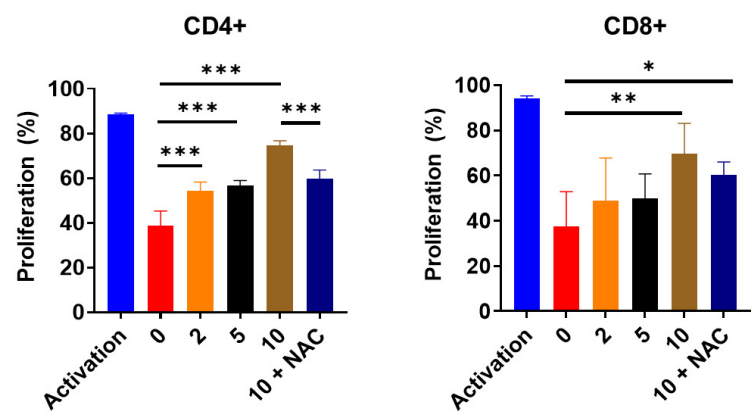

C

Total lymphocyte
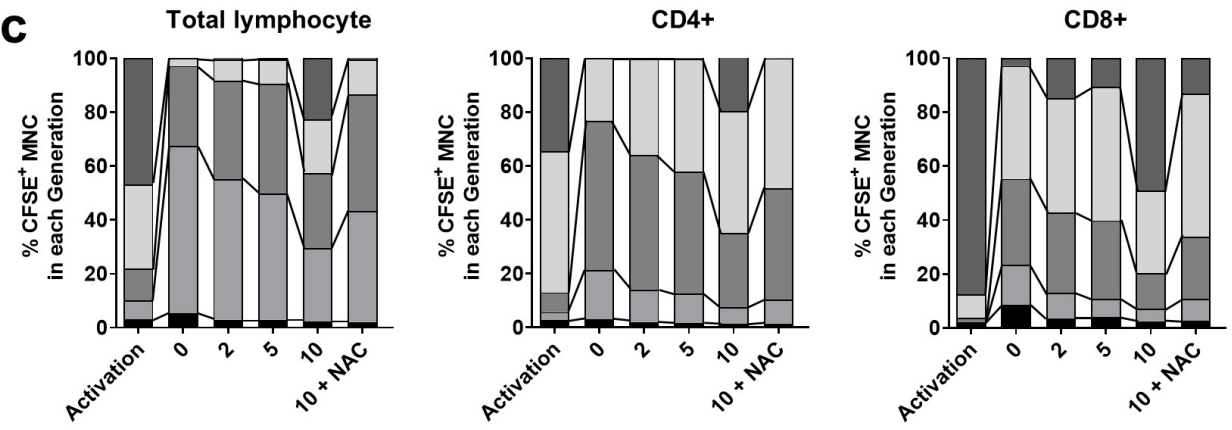

- 1st cell division $\square$ 2nd cell division

$\square$ 3rd cell divsion

$\square$ 4th cell division

5th cell division

d

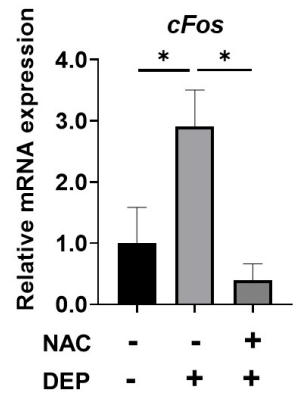

e

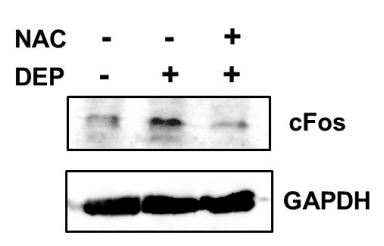

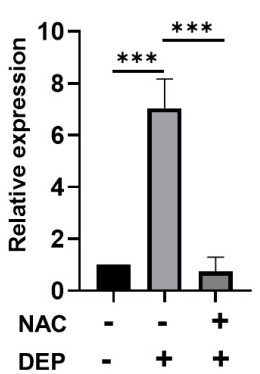

Fig. 3. DEP-induced ROS inhibits the immunomodulatory effects of WJ-MSCs. (a $\sim \mathrm{c})$ WJ-MSCs were pretreated for 30 min with NAC (5 $\mathrm{mM}$ ) and then were mitotically inactivated after treating with various concentrations of DEP for $48 \mathrm{hr}$. Cells were co-cultured with CFSE-labeled human mononuclear cells (MNCs) that were activated by CD3/CD28 Dynabeads and IL-2. After six days of co-culture, the percentage of $\mathrm{T}$ cell proliferation was measured using flow cytometry. (a) Representative histogram of total lymphocyte proliferation. (b) Proliferation of total, CD4 + T, and CD8 + T lymphocytes was quantified. (c) The number of cell divisions of total, CD4 + T, and CD8+ T lymphocytes was quantified based on dilution of CFSE. ( $d$, e) Cells were pretreated for $30 \mathrm{~min}$ with NAC and then were stimulated with $10 \mu \mathrm{g} / \mathrm{ml}$ DEP for $24 \mathrm{hr}$. (d) CFos mRNA was determined by qRT-PCR and normalized to $\beta$-actin mRNA. (e) cFos expression was quantified by western blot analysis. The data represent results from three independent experiments and are presented as mean $\pm \mathrm{SD}\left({ }^{*} \mathrm{p}<0.05 ;{ }^{* *} \mathrm{p}<0.01\right.$; $* * * \mathrm{p}<0.001)$.

creased the neutrophil infiltration (Fig. 6d and 6e). Histological examination showed that NAC group significantly rescued the submucosal thickening, the destruc- tion of the epithelium, infiltration of inflammatory cells (Fig. 6f). The NAC group also had reduced the histopathological scores compared with the DEP group (Fig. 6g). 

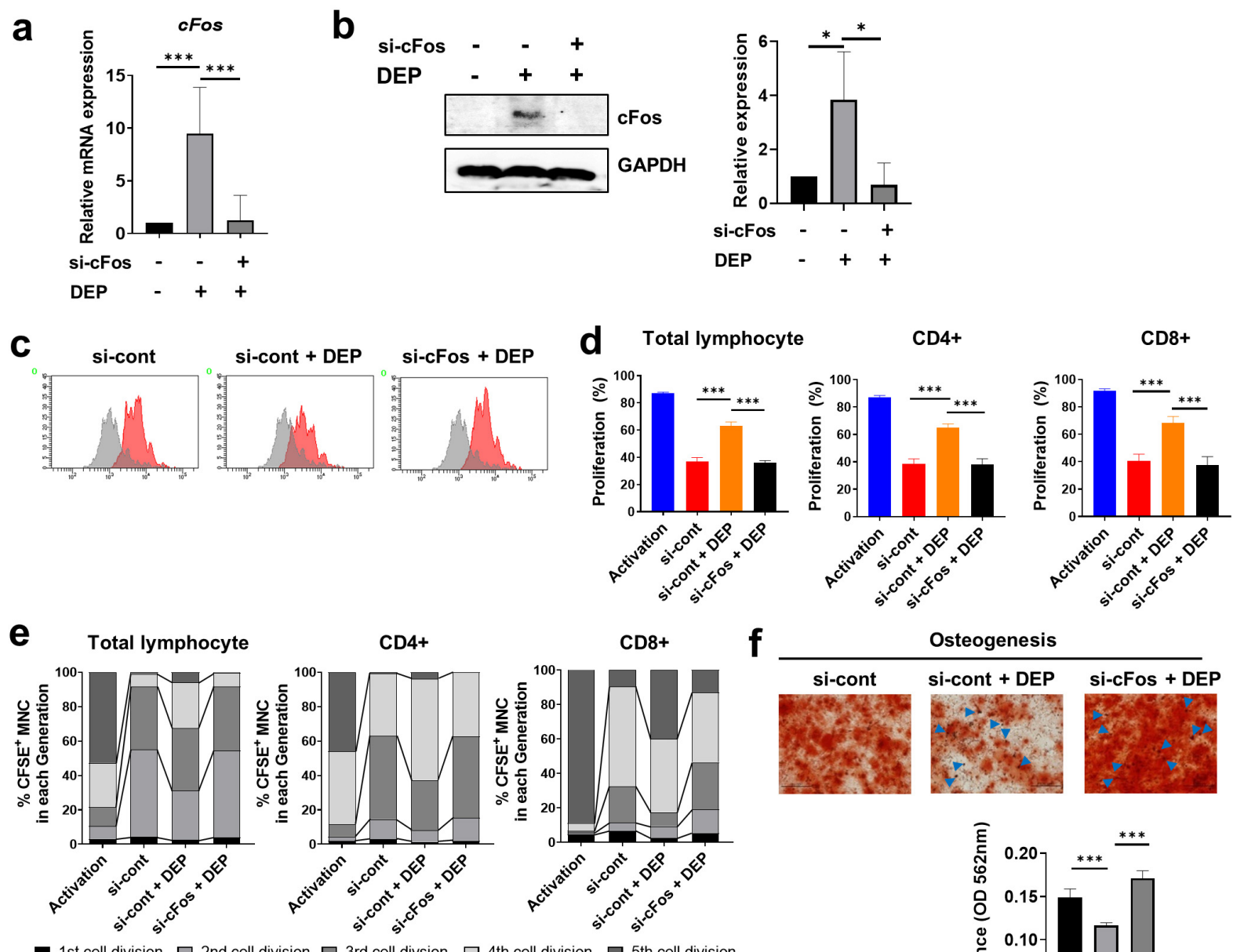

Osteogenesis

1st cell division $\square$ 2nd cell division $\square$ 3rd cell divsion $\square$ 4th cell division $\square 5$ th cell division
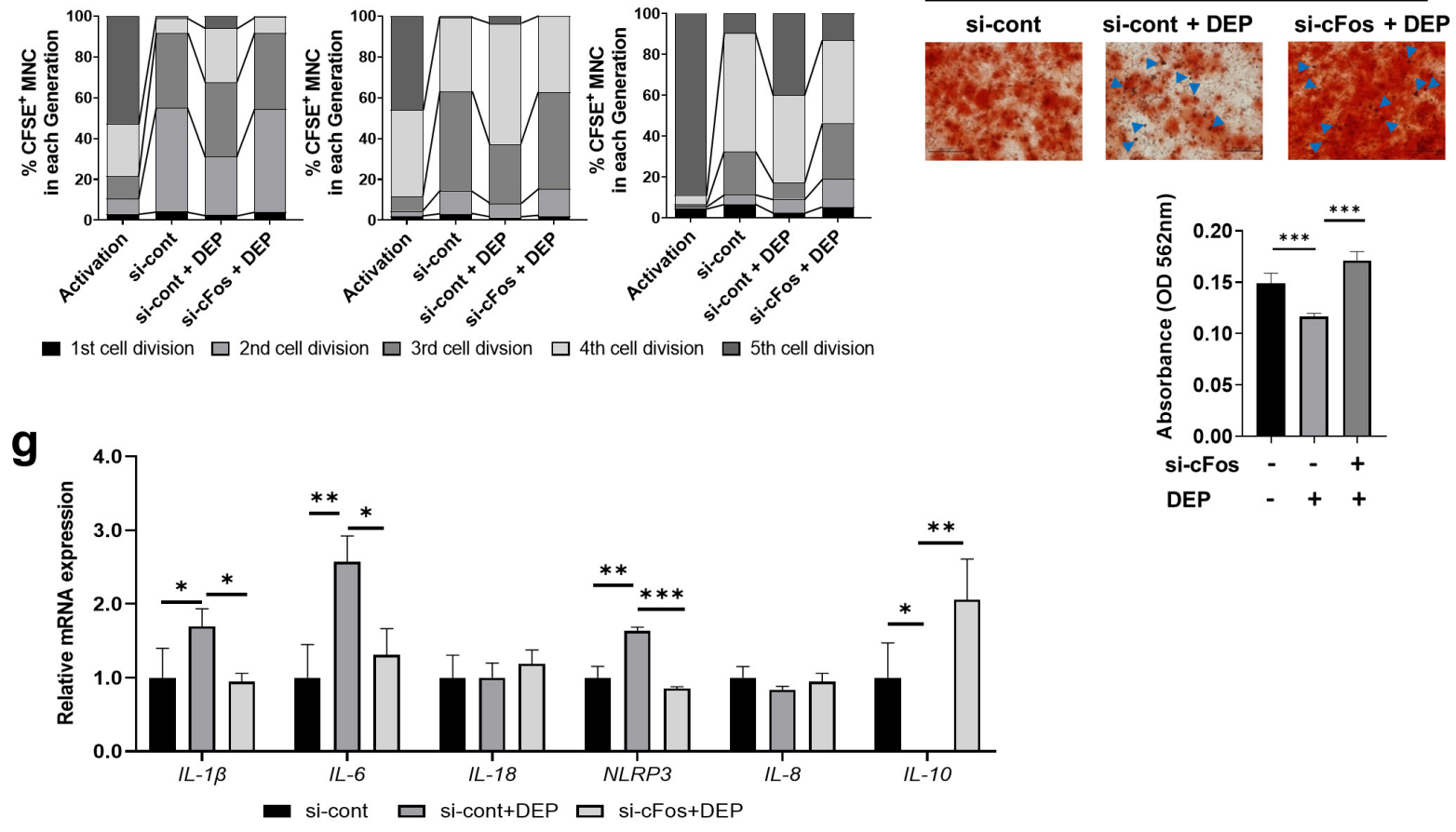

DEP -+++

Fig. 4. DEP-induced cFos expression inhibits the immunomodulatory function of WJ-MSCs. WJ-MSCs were transfected with control siRNA (si-cont) or CFos siRNA (si-cFos) for $4 \mathrm{hr}$ and then were stimulated with $10 \mu \mathrm{g} / \mathrm{ml}$ DEP. After $12 \mathrm{hr}$, knockdown of cFos was confirmed by qRT-PCR (a) and western blot (b). (c $\sim$ e) After $48 \mathrm{hr}$ of incubation, cells were mitotically inactivated and co-cultured with MNCs as previously described. (c) Representative histogram of total lymphocyte proliferation. (d) Proliferation of total, CD4 + T, and CD8 + T lymphocytes was quantified. (e) Number of cell divisions of total, CD4 $+\mathrm{T}$, and CD8 $+\mathrm{T}$ lymphocytes was quantified based on dilution of CFSE. (f) si-cFos-transfected WJ-MSCs were stimulated with DEP for $24 \mathrm{hr}$ and then cultured with osteogenic differentiation medium for 14 days. Calcium deposits were stained with Alizarin Red S. Light microscopy images of osteocytes show representative images from three independent experiments (Scale bar: $100 \mu \mathrm{m}$ ). Absorbance of calcium deposits was measured using a spectrophotometer. (g) Transcription of $I L-1 \beta, I L-6, I L-8, I L-18$, and NLRP3 was determined by qRT-PCR and normalized to $\beta$-actin transcription. Data are presented as mean \pm S.D. of triplicate experiments $(* \mathrm{p}<0.05 ; * * \mathrm{p}<0.01 ; * * * \mathrm{p}<0.001)$. 
a
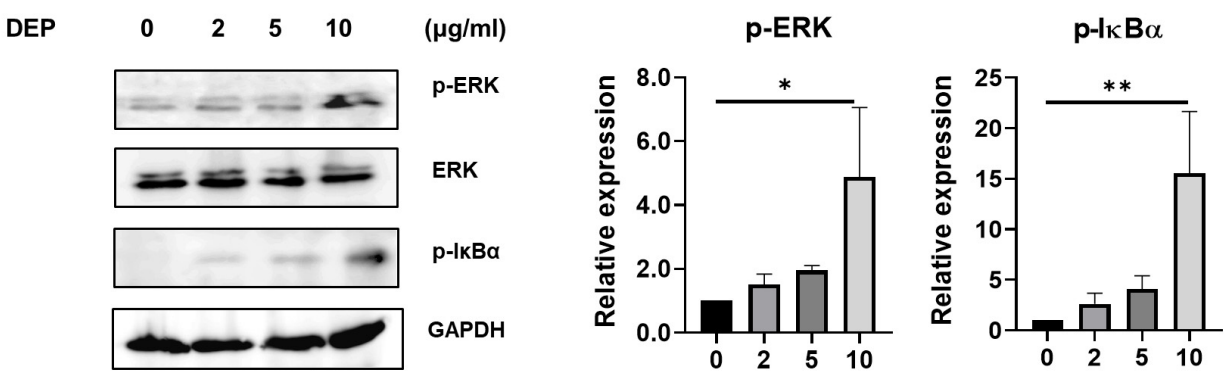

b
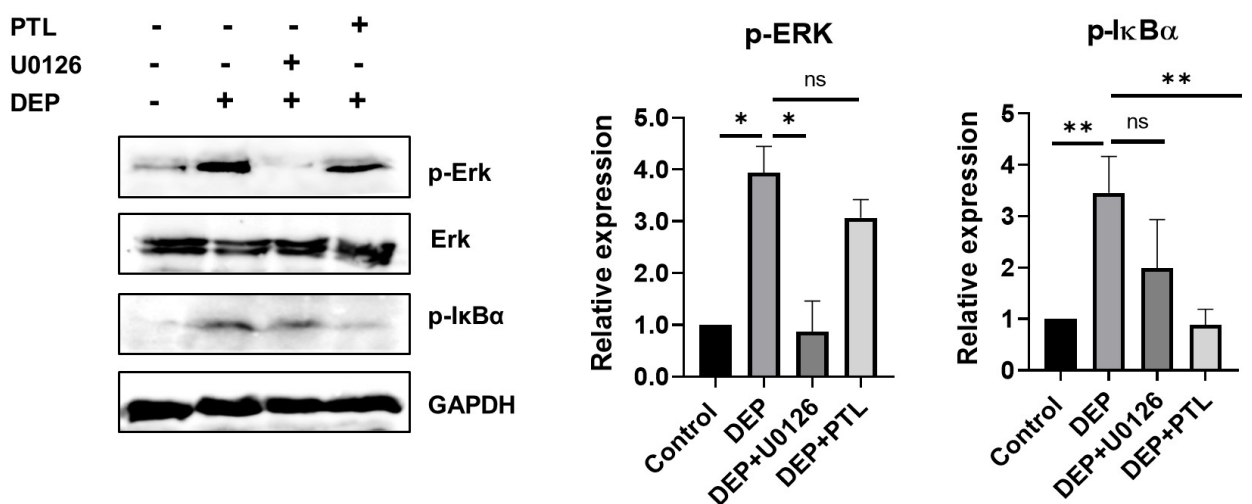

C
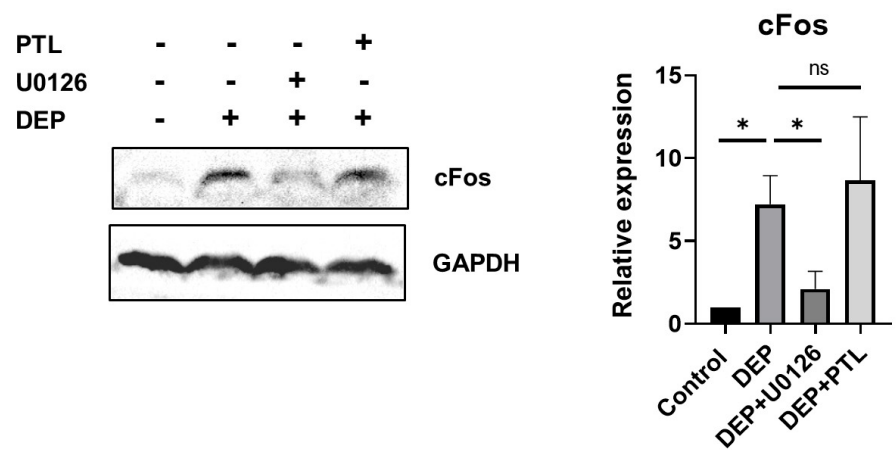

Fig. 5. DEP regulates cFos through ERK signaling. (a) WJ-MSCs were treated with DEP $(0 \sim 10 \mu \mathrm{g} / \mathrm{ml})$ for 1 hr. Activation of $p$-ERK and $\mathrm{p}-\mathrm{I} \kappa \mathrm{B} \alpha$ was determined by western blot analysis. (b, c) WJ-MSCs were pretreated with U0126 $(10 \mu \mathrm{M})$ or PTL $(5 \mu \mathrm{M})$ and then were treated with $10 \mu \mathrm{g} / \mathrm{ml}$ of DEP for $1 \mathrm{hr}$. Expression of p-ERK, $\mathrm{p}-\mathrm{l} \kappa \mathrm{B} \alpha$, and cFos was analyzed and quantified by western blotting (ns $>0.05$; $\left.{ }^{*} \mathrm{p}<0.05 ;{ }^{* *} \mathrm{p}<0.01\right)$.

Expression of pro-inflammatory cytokines, including $I F N^{-}$ $\gamma$ and $T N F a$ was decreased in colon tissue of NAC group compared to DEP group (Fig. 6h). Th1, Th17 and Treg, known to play an important role in the pathogenesis of inflammatory bowel disease, were analyzed in the colon of mice with experimental colitis. The DEP group significantly increased the expression levels of Th1 and Th17 lineage transcription factors compared to the WJ-MSC group, whereas the NAC group significantly decreased the expression levels of Th1 and Th17 transcription factors (Fig. 6i). However, Treg did not show any significant difference among groups. Taken together, these data indicate that DEP-induced ROS is involved in inhibiting the therapeutic effect of WJ-MSCs in DSS-induced colitis.

\section{Discussion}

Epidemiological studies have suggested that DEP exposure is associated with increased cardiovascular and respiratory morbidity and mortality. It has been suggested that DEP induce not only lung inflammation but also systemic damage of our body as lung inflammation possibly leak over to the cardiovascular system. Airborne PM were associated with dermatological, ocular, respiratory, car- 
a
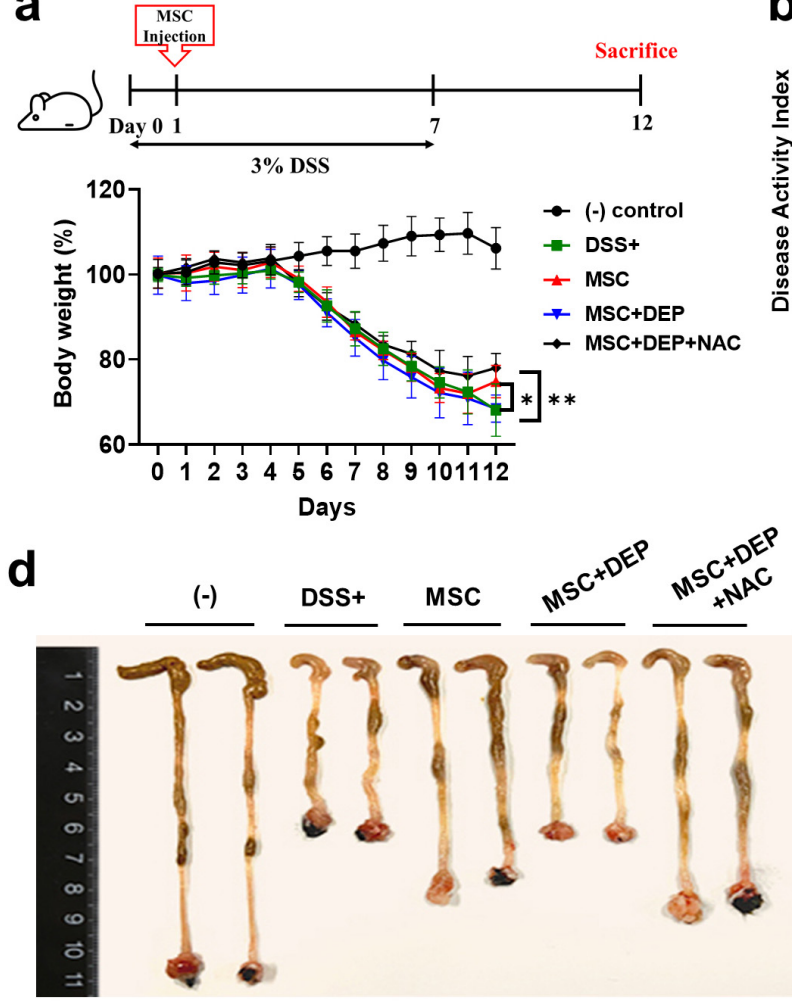

b

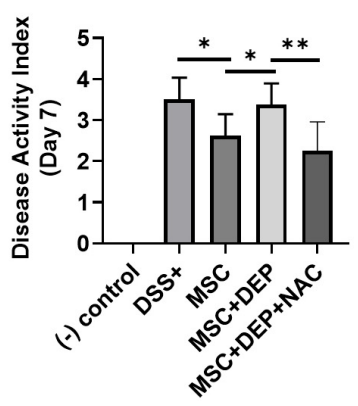

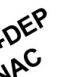

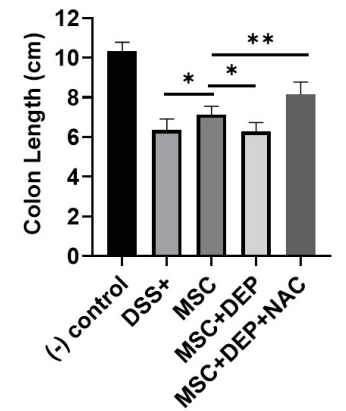

C

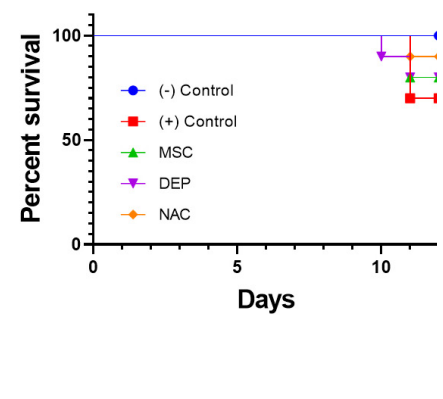

e

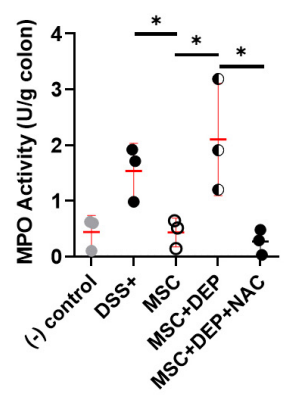

f

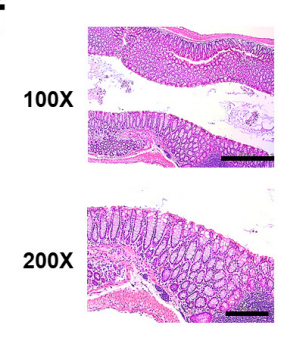

(-) control

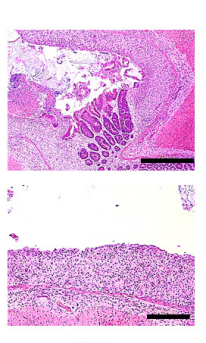

DSS+

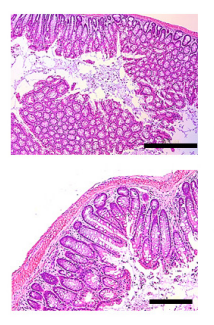

MSC

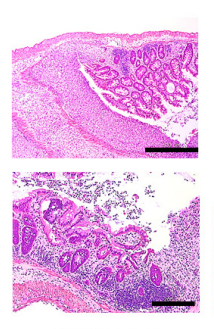

MSC+DEP

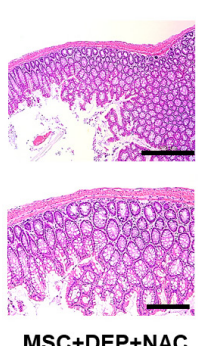

$M S C+D E P+N A C$ g

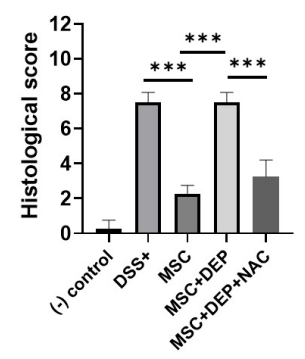

$\mathbf{h}$

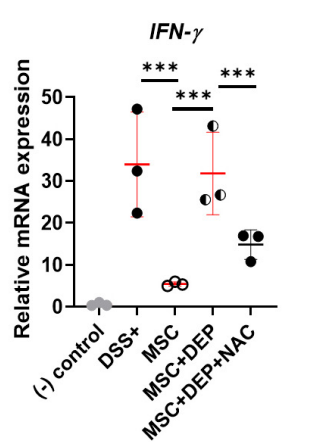

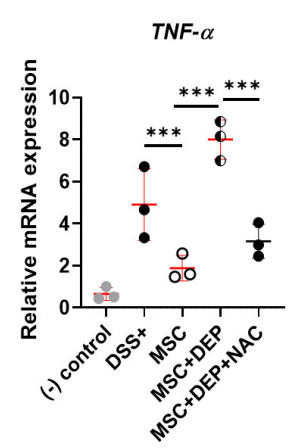

i

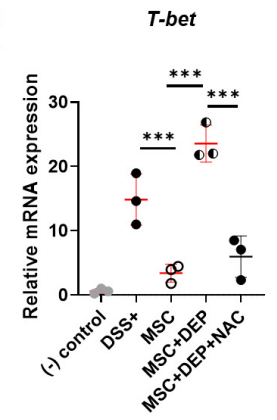

RORyt

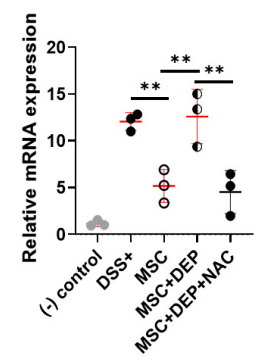

Foxp3

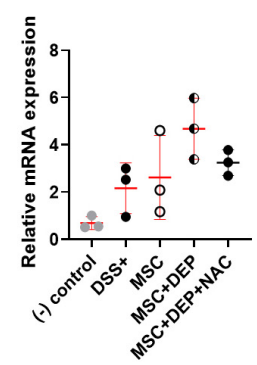

Fig. 6. DEP-induced ROS impairs the therapeutic effect of WJ-MSCs in colitis model. (a) The percentage of body weight change during entire experiment. (b) DAl scores were measured on day 7. (c) The survival rate of mice. (d) Mice were sacrificed on day 12, the length of colons was measured. (e) MPO activity was measured on day 12. (f) Colon tissue were examined using H\&E staining and (g) histological scores were shown. The scale bars represent $500 \mu \mathrm{m}$ and $200 \mu \mathrm{m}$. Expression levels of inflammatory cytokines (h) and Th1, Th17 and Treg associated cytokines (i) in colonic tissues were analyzed by qRT-PCR $\left(\mathrm{n}=3\right.$ mice per group; $\left.{ }^{*} \mathrm{p}<0.05 ;{ }^{*} \mathrm{p}<0.01 ;{ }^{* * *} \mathrm{p}<0.001\right)$. 
diovascular, neurological, immunological, metabolic diseases and cancer (22). Inhaled DEP possibly penetrate into the alveolar system; DEP binds to the protein transport system in pulmonary epithelial cells, passes into the circulatory system, and is transported to secondary target organs, such as the heart and liver, where it causes inflammation (25). Previous studies have shown that DEP induces ROS generation in endothelial cells exposed to high concentrations of DEP, which leads to cellular apoptosis with Mdm2 depletion (26). In addition, WJ-MSCs might modulate endothelial responses such as leukocyte recruitment or vascular inflammatory responses (27). Although DEP has been reported to induce inflammation and apoptosis of human bone marrow (BM)-derived MSCs (22), the exact mechanism of the interactions between DEP and WJ-MSCs has not been elucidated. In light of previous studies indicating that PAHs in DEP enhance oxidative stress and activate pro-inflammatory responses in macrophages, bronchial epithelial cells, and naïve $\mathrm{T}$ cells, we evaluated the direct effect of DEP on the immunomodulatory function of WJ-MSCs $(19,23,28)$.

Activation of NF- $\kappa \mathrm{B}$ and expression of $\mathrm{cFos}$ by DEP exposure have been reported to contribute to the proliferation and pro-inflammatory processes of human bronchial epithelial cells (23). In the present study, we used RNA sequencing to identify 504 genes with markedly altered expressions in DEP-treated WJ-MSCs, and the most enriched gene set was that for metabolic processes, including cFos. DEP-induced effects in the lungs have been reported to involve cFos, but the possible relationship between regulation of pro-inflammatory mediators and $\mathrm{cFos}$ has not been thoroughly investigated (29). We demonstrated that DEPs induced pronounced expression of $\mathrm{cFos}$ and significantly increased the expression of pro-inflammatory factors such as $I F N \gamma, I L-1 \beta, I L-6$, and NLRP3. Our findings suggest that $\mathrm{cFos}$ plays an important role in activation of DEP-induced NLRP3 inflammasome signals, since the expression of pro-inflammatory cytokines was almost abolished by inhibition of cFos.

Indeed, DEP has been reported to induce ROS, which are involved in cytokine formation, cytotoxicity, and DNA damage $(1,22)$. In addition, ROS can reduce the immunomodulatory functions of WJ-MSCs and promote their senescence and inflammation (30). In the present study, we confirmed that DEP increases ROS production in WJ-MSCs. In addition, DEP exposure inhibited the immunosuppressive effect of WJ-MSCs on $\mathrm{T}$ cell proliferation, and this response was abolished by pretreatment with the anti-oxidant NAC. Furthermore, WJ-MSCs incubated with DEP impaired the therapeutic effect in the experimental colitis mice and increased Th1 and Th17 cell responses, which were also abolished by the antioxidant NAC pretreatment. Our results indicate that DEP inhibits the immunosuppressive effects of WJ-MSCs by modulating ROS production. Although the exact mechanism of DEP-induced immune cell activation has not been studied thoroughly, it has been suggested that DEP increase bronchial hypersensitivity in asthmatic patients by enhancing basal $\mathrm{T}$ cell activation $(2,31)$. In the current study, we found that DEP did not directly affect $\mathrm{T}$ cell proliferation (Supplementary Fig. 3c) but inhibited WJ-MSC-mediated suppression of $\mathrm{T}$ cell proliferation. The antioxidant NAC inhibited the DEP-induced increase in cFos expression, suggesting that stimulation of cFos likely is dependent on activation of ROS. In addition, cFos inhibits osteogenesis and adipogenesis in immortalized human MSC progenitor cells (32). Our findings suggest that cFos activation is important for DEP inhibition of the osteogenic differentiation of WJ-MSCs because inhibition of their differentiation was reversed by cFos inhibition.

Exposure to DEP and the consequent oxidative stress have been shown to activate redox-sensitive transcription factors and various signal transduction pathways, including $\mathrm{AP}-1, \mathrm{NF}-\kappa \mathrm{B}, \mathrm{MAPKs}, \mathrm{p} 38$, and JNK in airway epithelial cells (24). Activation of these proteins promotes the transcription of proinflammatory mediators, triggering the characteristic pulmonary inflammatory response of DEP exposure (33). We detected DEP-induced activation of p-I $\kappa \mathrm{Ba}$ and $\mathrm{p}$-ERK; however, DEP-induced $\mathrm{cFos}$ expression was only reduced by ERK inhibition and was not affected by NF- $\kappa$ B inhibition. Similarly, DEP can induce release of pro-inflammatory cytokines through NF- $\kappa \mathrm{B}$ or $\mathrm{AP}-1$ regulatory pathways (34). Although no direct immunomodulatory function analysis of $\mathrm{NF}-\kappa \mathrm{B}$ was performed in this study, NF- $\kappa \mathrm{B}$ activation induced by DEP could be attributed to stimulation of immune system responses; however, further study is needed to address this hypothesis.

It has recently been reported that atmospheric PM causes inflammation in lung cells, and that exposure to PM can increase the sensitivity and severity of symptoms in COVID-19 patients (35). Furthermore, air pollutants induce angiotensin converting enzyme 2 (ACE2) receptor expression in human epithelial cells $(36,37)$. Because WJ-MSCs have the potential to suppress immune system hyperactivity and inhibit cytokine storms with low expression of ACE2, COVID-19 patients were administered WJ-MSCs in a recent COVID-19 clinical trial $(38,39)$. Our study showed that DEP significantly impaired the 
immunomodulatory properties of WJ-MSCs; future studies of DEP-ACE2 interactions can inform potential therapeutic approaches for COVID-19 based upon WJ-MSCs.

Here we provide evidence that exposure to DEP enhances the expression of pro-inflammatory cytokines and suppresses the immunomodulatory properties through mechanisms involving ROS/ERK/cFos pathway in WJ-MSCs. In addition, we confirmed that DEP-induced ROS damage impairs the therapeutic efficacy of WJ-MSCs against inflammatory bowel disease, using DSS-induced colitis model. Therefore, exposure to DEP can affect the regenerative and immunomodulatory properties of WJ-MSCs and influence the pathophysiology of DEP-related diseases. Modulation of $\mathrm{ROS} / \mathrm{ERK} / \mathrm{cFos}$ signaling pathways in WJ-MSCs could be a novel target for acute and chronic inflammatory processes induced by DEP exposure.

\section{Acknowledgments}

This research was supported by the Basic Research Program through the National Research Foundation of Korea (NRF) (2019R1C1C1008896) funded by the Korean government.

\section{Potential Conflict of Interest}

The authors have no conflicting financial interest.

\section{Supplementary Materials}

Supplementary data including one table and four figures can be found with this article online at https://doi.org/ $10.15283 / \mathrm{ijsc} 21178$.

\section{References}

1. Wilson SJ, Miller MR, Newby DE. Effects of diesel exhaust on cardiovascular function and oxidative stress. Antioxid Redox Signal 2018;28:819-836

2. Li N, Hao M, Phalen RF, Hinds WC, Nel AE. Particulate air pollutants and asthma. A paradigm for the role of oxidative stress in PM-induced adverse health effects. Clin Immunol 2003;109:250-265

3. van Eeden SF, Tan WC, Suwa T, Mukae H, Terashima T, Fujii T, Qui D, Vincent R, Hogg JC. Cytokines involved in the systemic inflammatory response induced by exposure to particulate matter air pollutants (PM(10)). Am J Respir Crit Care Med 2001;164:826-830

4. Totlandsdal AI, Cassee FR, Schwarze P, Refsnes M, Låg $M$. Diesel exhaust particles induce CYP1A1 and pro-inflammatory responses via differential pathways in human bronchial epithelial cells. Part Fibre Toxicol 2010;7:41

5. Quintana FJ, Basso AS, Iglesias AH, Korn T, Farez MF,
Bettelli E, Caccamo M, Oukka M, Weiner HL. Control of $\mathrm{T}(\mathrm{reg})$ and $\mathrm{T}(\mathrm{H}) 17$ cell differentiation by the aryl hydrocarbon receptor. Nature 2008;453:65-71

6. Yu KR, Kang KS. Aging-related genes in mesenchymal stem cells: a mini-review. Gerontology 2013;59:557-563

7. Jimenez-Puerta GJ, Marchal JA, López-Ruiz E, GálvezMartín P. Role of mesenchymal stromal cells as therapeutic agents: potential mechanisms of action and implications in their clinical use. J Clin Med 2020;9:445

8. Yu KR, Lee JY, Kim HS, Hong IS, Choi SW, Seo Y, Kang I, Kim JJ, Lee BC, Lee S, Kurtz A, Seo KW, Kang KS. A p38 MAPK-mediated alteration of COX-2/PGE2 regulates immunomodulatory properties in human mesenchymal stem cell aging. PLoS One 2014;9:e102426

9. Song N, Scholtemeijer M, Shah K. Mesenchymal stem cell immunomodulation: mechanisms and therapeutic potential. Trends Pharmacol Sci 2020;41:653-664

10. Kang JY, Oh MK, Joo H, Park HS, Chae DH, Kim J, Lee $\mathrm{HR}$, Oh IH, Yu KR. Xeno-free condition enhances therapeutic functions of human Wharton's jelly-derived mesenchymal stem cells against experimental colitis by upregulated indoleamine 2,3-dioxygenase activity. J Clin Med 2020;9:2913

11. Joo H, Oh MK, Kang JY, Park HS, Chae DH, Kim J, Lee JH, Yoo HM, Choi U, Kim DK, Lee H, Kim S, Yu KR. Extracellular vesicles from thapsigargin-treated mesenchymal stem cells ameliorated experimental colitis via enhanced immunomodulatory properties. Biomedicines 2021; 9:209

12. Fontaine MJ, Shih H, Schäfer R, Pittenger MF. Unraveling the mesenchymal stromal cells' paracrine immunomodulatory effects. Transfus Med Rev 2016;30:37-43

13. Kim SY, Oh SH, Lee JH, Suh MW, Park MK. Effects of placenta-derived mesenchymal stem cells on the particulate matter-induced damages in human middle ear epithelial cells. Stem Cells Int 2019;2019:4357684

14. Munir H, McGettrick HM. Mesenchymal stem cell therapy for autoimmune disease: risks and rewards. Stem Cells Dev 2015;24:2091-2100

15. Trapnell C, Pachter L, Salzberg SL. TopHat: discovering splice junctions with RNA-Seq. Bioinformatics 2009;25: 1105-1111

16. Trapnell C, Williams BA, Pertea G, Mortazavi A, Kwan G, van Baren MJ, Salzberg SL, Wold BJ, Pachter L. Transcript assembly and quantification by RNA-Seq reveals unannotated transcripts and isoform switching during cell differentiation. Nat Biotechnol 2010;28:511-515

17. Trapnell C, Roberts A, Goff L, Pertea G, Kim D, Kelley DR, Pimentel H, Salzberg SL, Rinn JL, Pachter L. Differential gene and transcript expression analysis of RNA-seq experiments with TopHat and Cufflinks. Nat Protoc 2012;7:562-578

18. Benjamini Y, Hochberg Y. Controlling the false discovery rate: a practical and powerful approach to multiple testing. J R Stat Soc Ser B 1995;57:289-300

19. O'Driscoll CA, Owens LA, Gallo ME, Hoffmann EJ, Afrazi 
A, Han M, Fechner JH, Schauer JJ, Bradfield CA, Mezrich JD. Differential effects of diesel exhaust particles on $\mathrm{T}$ cell differentiation and autoimmune disease. Part Fibre Toxicol 2018;15:35

20. Meldrum K, Gant TW, Leonard MO. Diesel exhaust particulate associated chemicals attenuate expression of CXCL10 in human primary bronchial epithelial cells. Toxicol In Vitro 2017;45(Pt 3):409-416

21. Raudoniute J, Stasiulaitiene I, Kulvinskiene I, Bagdonas E, Garbaras A, Krugly E, Martuzevicius D, Bironaite D, Aldonyte R. Pro-inflammatory effects of extracted urban fine particulate matter on human bronchial epithelial cells BEAS-2B. Environ Sci Pollut Res Int 2018;25:32277-32291

22. Abu-Elmagd M, Alghamdi MA, Shamy M, Khoder MI, Costa M, Assidi M, Kadam R, Alsehli H, Gari M, Pushparaj PN, Kalamegam G, Al-Qahtani MH. Evaluation of the effects of airborne particulate matter on bone marrow-mesenchymal stem cells (BM-MSCs): cellular, molecular and systems biological approaches. Int J Environ Res Public Health 2017;14:440

23. Baeza-Squiban A, Bonvallot V, Boland S, Marano F. Diesel exhaust particles increase NF-kappaB DNA binding activity and c-FOS proto-oncogene expression in human bronchial epithelial cells. Toxicol In Vitro 1999;13:817-822

24. Pourazar J, Mudway IS, Samet JM, Helleday R, Blomberg A, Wilson SJ, Frew AJ, Kelly FJ, Sandström T. Diesel exhaust activates redox-sensitive transcription factors and kinases in human airways. Am J Physiol Lung Cell Mol Physiol 2005;289:L724-L730

25. Donaldson K, Tran L, Jimenez LA, Duffin R, Newby DE, Mills N, MacNee W, Stone V. Combustion-derived nanoparticles: a review of their toxicology following inhalation exposure. Part Fibre Toxicol 2005;2:10

26. Tseng CY, Wang JS, Chang YJ, Chang JF, Chao MW. Exposure to high-dose diesel exhaust particles induces intracellular oxidative stress and causes endothelial apoptosis in cultured in vitro capillary tube cells. Cardiovasc Toxicol 2015;15:345-354

27. Luu NT, McGettrick HM, Buckley CD, Newsome PN, Rainger GE, Frampton J, Nash GB. Crosstalk between mesenchymal stem cells and endothelial cells leads to downregulation of cytokine-induced leukocyte recruitment. Stem Cells 2013;31:2690-2702

28. Vogel CF, Sciullo E, Wong P, Kuzmicky P, Kado N, Matsumura F. Induction of proinflammatory cytokines and C-reactive protein in human macrophage cell line U937 exposed to air pollution particulates. Environ Health Perspect 2005;113:1536-1541
29. Li R, Kou X, Xie L, Cheng F, Geng H. Effects of ambient PM2.5 on pathological injury, inflammation, oxidative stress, metabolic enzyme activity, and expression of c-fos and c-jun in lungs of rats. Environ Sci Pollut Res Int 2015;22:20167-20176

30. Lee BC, Yu KR. Impact of mesenchymal stem cell senescence on inflammaging. BMB Rep 2020;53:65-73

31. Mamessier E, Nieves A, Vervloet D, Magnan A. Diesel exhaust particles enhance T-cell activation in severe asthmatics. Allergy 2006;61:581-588

32. Abarrategi A, Gambera S, Alfranca A, Rodriguez-Milla MA, Perez-Tavarez R, Rouault-Pierre K, Waclawiczek A, Chakravarty P, Mulero F, Trigueros C, Navarro S, Bonnet D, García-Castro J. c-Fos induces chondrogenic tumor formation in immortalized human mesenchymal progenitor cells. Sci Rep 2018;8:15615

33. Baulig A, Garlatti M, Bonvallot V, Marchand A, Barouki $\mathrm{R}$, Marano F, Baeza-Squiban A. Involvement of reactive oxygen species in the metabolic pathways triggered by diesel exhaust particles in human airway epithelial cells. Am J Physiol Lung Cell Mol Physiol 2003;285:L671-L679

34. Tal TL, Simmons SO, Silbajoris R, Dailey L, Cho SH, Ramabhadran R, Linak W, Reed W, Bromberg PA, Samet JM. Differential transcriptional regulation of IL-8 expression by human airway epithelial cells exposed to diesel exhaust particles. Toxicol Appl Pharmacol 2010;243:46-54

35. Hendryx M, Luo J. COVID-19 prevalence and fatality rates in association with air pollution emission concentrations and emission sources. Environ Pollut 2020;265(Pt A): 115126

36. Baulig A, Blanchet S, Rumelhard M, Lacroix G, Marano F, Baeza-Squiban A. Fine urban atmospheric particulate matter modulates inflammatory gene and protein expression in human bronchial epithelial cells. Front Biosci 2007;12:771-782

37. Paital B, Agrawal PK. Air pollution by $\mathrm{NO}_{2}$ and $\mathrm{PM}_{2.5}$ explains COVID-19 infection severity by overexpression of angiotensin-converting enzyme 2 in respiratory cells: a review. Environ Chem Lett 2021;19:25-42

38. Avanzini MA, Mura M, Percivalle E, Bastaroli F, Croce S, Valsecchi C, Lenta E, Nykjaer G, Cassaniti I, Bagnarino J, Baldanti F, Zecca M, Comoli P, Gnecchi M. Human mesenchymal stromal cells do not express ACE2 and TMPRSS2 and are not permissive to SARS-CoV-2 infection. Stem Cells Transl Med 2021;10:636-642

39. Lee BC, Kang I, Yu KR. Therapeutic features and updated clinical trials of mesenchymal stem cell (MSC)-derived exosomes. J Clin Med 2021;10:711 